\title{
Perfil e produção científica dos bolsistas de produtividade em pesquisa do CNPQ da área de estomaterapia
}

\author{
Profile and production of scientific researchers with \\ productivity grants in stomatherapy of CNPQ
}

\begin{abstract}
Tamires Barradas Cavalcante ${ }^{1} \bullet$ Moniki de Oliveira Barbosa Campos ${ }^{2} \bullet$ Ana Karine da Costa Monteiro ${ }^{3}$ Elaine Maria Leite Rangel Andrade ${ }^{4}$ Jesusmar Ximenes Andrade ${ }^{5}$
\end{abstract}

\section{RESUMO}

Este estudo teve como objetivo identificar o perfil e a produção científica dos bolsistas produtividade em pesquisa do CNPq da área de Estomaterapia no triênio 2010-2012. Estudo descritivo, transversal e quantitativo. Para a coleta de dados, no mês de maio de 2014, preencheu-se a partir dos currículos Lattes, um instrumento sobre: sexo, estado de procedência, instituição de formação, categoria administrativa de atuação, tempo de formação, tempo de doutoramento, categoria profissional, classificação da bolsa, produção científica de artigos publicados em periódicos e seus respectivos Qualis. Foram identificados 16 bolsistas e a maioria, 12 (75\%), era do sexo feminino e da região sudeste, 9 (56,3\%). 0 tempo médio de formação e de doutoramento foi respectivamente 33,3 e 16,4 anos. Quanto à instituição de formação e a categoria administrativa da instituição prevaleceu a estadual, respectivamente, 8 (50\%) e 11 (68,8\%). Entre os bolsistas, predominou a Enfermagem, 13 (81,3\%), e bolsas PQ-2, 8 (50\%). Em média, os bolsistas publicaram 24,7 ( $D P=14,9)$ artigos sobre temáticas gerais e 2,5 $(D P=5,3)$ na área de Estomaterapia. Estudos dessa natureza são necessários para verificar o que tem sido produzido pela Estomaterapia.

Palavras-chave: Indicadores de produção científica; Enfermagem; Indicadores bibliométricos.

\begin{abstract}
This study aimed to identify the profile and scientific production of researchers with productivity grants of CNPq in stomatherapy in the 2010-2012 triennium. Descriptive, cross-sectional quantitative study. Data collection was realized through the lattes curriculum in may 2014,from filling out an instrument about: gender, state of origin, training institution, formation time, doctoral time, professional category, classification of grant, scientific production of published journals and their respective Qualis. Were identified 16 recipients of grants and most of 12 (75\%) was female and of the southeast region (56.3\%). Average training and doctoral time was respectively 33.31 and 16.44 years. As training institution and the administrative category of institution prevailed statewide, respectively 8 (50\%) and 11 (68.8\%). Among the recipients of grants, prevailed nursing 13 (81.3\%) and grant 2 category (50\%). In average the grants' recipients published $24,7(S D=14,9)$ articles about general topics and 2,5 $(S D=5,3)$ in stomatherapy area. Such studies are necessary to verify what has been produced in stomathrerapy.
\end{abstract}

Keywords: Scientific production indicators; Nursing; Bibliometric indicators.

${ }^{1}$ Mestre em Enfermagem pela Universidade Federal do Piauí (UFPI).Teresina, Piauí, Brasil. E-mail: tamiresbarradas@gmail.com

2 Mestre em Enfermagem pela Universidade Federal do Piauí (UFPI). Teresina, Piauí, Brasil. E-mail: monikibarbosa@hotmail.com

${ }^{3}$ Mestre em Enfermagem pela Universidade Federal do Piauí (UFPI).Teresina, Piauí, Brasil. E-mail: karinemonteiro2006@hotmail.com

${ }^{4}$ Doutora em Enfermagem pela Escola de Enfermagem de Ribeirão Preto da Universidade de São Paulo (EERP-USP). Docente da Universidade Federal do Piauí - (UFPI). Teresina, Piauí, Brasil. E-mail: elairgel@gmail.com

${ }^{5}$ Doutor pela Faculdade de Economia, Administração e Contabilidade da Universidade de São Paulo - FEA/USP. Docente da Universidade Federal do Piauí - (UFPI). Teresina, Piauí, Brasil. E-mail: jesusmar.andrade@gmail.com

Autor Responsável: Tamires Barradas Cavalcante.E-mail: tamiresbarradas@gmail.com 


\section{INTRODUÇÃO}

No Brasil, a produção científica está em expansão, em 2002 o país ocupava o $17^{\circ}$ lugar do Ranking mundial em número de artigos científicos publicados e em 2012 passou a ser o $13^{\circ}$ lugar. Na América Latina, o Brasil lidera o Ranking, com 53.083 artigos científicos publicados em 2012 pelos pesquisadores brasileiros ${ }^{1}$.

Vários fatores podem explicar essa expansão, entre eles a criação do Conselho Nacional de Pesquisa (CNPq), na década de 50, que possibilitou formação de pesquisadores e as bolsas produtividade em pesquisa (PO), criadas em 1976, que valorizam aqueles pesquisadores que se destacam pela sua produção científica. 0 perfil de bolsistas produtividade em pesquisa tem interessado a comunidade cientifica ${ }^{2-3}$.

$\mathrm{Na}$ literatura nacional, observa-se preocupação quanto à caracterização dos bolsistas e sua produção científica em diversas áreas da saúde. Na área médica, pesquisadores de Nefrologia e Urologia, bem como da Pediatria, analisaram os bolsistas produtividade do $\mathrm{CNPq}$ quanto às variáveis: sexo, instituição, tempo de doutoramento, artigos publicados e orientação de alunos de graduação, mestrado e doutorado ${ }^{4-5}$. Na Odontologia, pesquisa semelhante analisou o perfil dos bolsistas também segundo critérios de classificação no CNPq (1A a 1D e 2) e quantidade de artigos publicados com os respectivos Qualis, livros e capítulos de Livros ${ }^{6}$. Na Enfermagem, estudo do perfil de bolsistas produtividade em pesquisa do $\mathrm{CNPq}$ verificou número reduzido desses pesquisadores, principalmente quando comparados ao número de bolsas por região do país e por estados. As variáveis do estudo estavam relacionadas à caracterização sociodemográfica e curricular dos bolsistas ${ }^{7}$.

A Estomaterapia é uma especialidade jovem, nascida em 1961, nos Estados Unidos da América. No Brasil, somente em 1990 o primeiro curso foi instituído. Em formato de pós-graduação latu sensu ou extensão da enfermagem, consiste no estudo do cuidado a pessoas com feridas agudas, estomas e incontinências ${ }^{8}$.

$\mathrm{Na}$ área de Estomaterapia, não foram encontradas pesquisas sobre o perfil e produção científica dos bolsistas produtividade em pesquisa. Esse tipo de pesquisa pode configurar uma ferramenta importante ao permitir entender, observar e analisar as tendências e perspectivas da produção científica nessa área, considerada jovem.

Diante disso, o objetivo deste estudo foi identificar o perfil e a produção científica dos bolsistas produtividade em pesquisa do CNPq da área de Estomaterapia.

\section{METODOLOGIA}

Trata-se de estudo descritivo, transversal e quantitativo, realizado no mês de maio de 2014.

Fizeram parte do estudo todos os $(n=16)$ bolsistas produtividade em pesquisa do $\mathrm{CNPq}$ da área de
Estomaterapia, com bolsas ativas no triênio 2010-2012. Para localizá-los foi realizada busca por assunto dos currículos na plataforma Lattes utilizando a palavra-chave "Estomaterapia", com filtro para bolsistas produtividade do CNPq. Essa plataforma é um "conjunto de sistemas computacionais do CNPq que compatibiliza e integra as informações em toda interação da Agência com seus usuários" e seu objetivo é aprimorar a qualidade dessas informações e racionalizar o trabalho de estudantes e pesquisadores no seu preenchimento, nela é possível acessar o Currículo Lattes, indispensável aos pesquisadores 9

Para coleta de dados, preencheu-se, a partir dos currículos Lattes, um instrumento sobre: sexo, estado de procedência, instituição de formação, categoria administrativa de atuação, tempo de formação, tempo de doutoramento, categoria profissional, classificação da bolsa, produção científica de artigos publicados em periódicos e seus respectivos Qualis.

De posse das informações, foi construído um dicionário de dados e realizada dupla digitação em planilhas do aplicativo Microsoft Excel. Após, os erros foram corrigidos pelo processo de validação e os dados exportados e analisados no software SPSS (Statistical Package for Social Science) versão 18.0. Estatísticas descritivas foram realizadas do tipo: média, desvio padrão, frequência absoluta e percentual. Os resultados apresentados por meio de tabelas e gráficos.

\section{RESULTADOS}

$\mathrm{Na}$ Tabela 1 está presente a análise descritiva dos dados sociodemográficos e de formação dos participantes do estudo. Foram identificados dezesseis bolsistas produtividade em pesquisa do CNPq da área de Estomaterapia no triênio de 2010-2012 e a maioria era do sexo feminino, 12 (75\%), e da região sudeste, 9 (56,3\%). O tempo médio de formação e de doutoramento foi respectivamente 33,3 e 16,4 anos. Quanto à instituição de formação e a categoria administrativa da instituição prevaleceu a estadual, respectivamente, 8 (50\%) e 11 $(68,8 \%)$. Entre os bolsistas, predominou a categoria profissional de Enfermagem, 13 (81,3\%), e bolsas PQ-2, 8 (50\%) (Tabela 1).

Em média, os bolsistas produtividade em pesquisa publicaram 24,7 ( $D P=14,9)$ artigos sobre temáticas gerais e 2,5 $(D P=5,3)$ na área de Estomaterapia em periódicos Qualis A2 7,2 (DP=5,6), B2 5,8 (DP=4,9) e B1 4,2 (DP=4,9).

A Figura 1 ilustra a relação entre os Qualis dos periódicos cujas pesquisas dos bolsistas de produtividade foram publicadas e as suas respectivas categorias de bolsa. Entre os bolsistas produtividade em pesquisa das categorias de bolsa 1C e 1B prevaleceu o Qualis A1 e A2 (Figura 1). 
Tabela 1. Perfil sociodemográico e profissional dos bolsistas produtividade em pesquisa do $\mathrm{CNPq}$ na área de Estomaterapia triênio 2010-2012. Teresina, PI, Brasil, 2014.

\begin{tabular}{|c|c|c|c|c|}
\hline CARACTERÍSTICAS & $\bar{x}^{\mathrm{i}}(s)^{\mathrm{ii}}$ & $\begin{array}{c}\min - \\
\max \end{array}$ & $n$ & $\%$ \\
\hline \multicolumn{5}{|l|}{ Sexo } \\
\hline Masculino & & & 4 & 25,0 \\
\hline Feminino & & & 12 & 75,0 \\
\hline \multicolumn{5}{|l|}{ Região } \\
\hline Sudeste & & & 9 & 56,3 \\
\hline Nordeste & & & 5 & 31,3 \\
\hline Sul & & & 2 & 12,5 \\
\hline Tempo de Formação & $\begin{array}{l}33,3 \\
(8,1)\end{array}$ & $17-44$ & - & - \\
\hline Tempo de Doutoramento & $\begin{array}{l}16,4 \\
(3,6)\end{array}$ & $11-21$ & - & - \\
\hline
\end{tabular}

\begin{tabular}{l|l|l|l|l}
\hline Instituição de Formação & \multicolumn{5}{|l}{} \\
\hline Federal & & & 5 & 31,3 \\
\hline Estadual & & & 8 & 50,0 \\
\hline Particular & & & 3 & 18,8 \\
\hline
\end{tabular}

\begin{tabular}{l|c|c|c|c}
\hline \multicolumn{3}{l}{ Categoria administrativa da instituição } \\
\hline Federal & & & 4 & 25,0 \\
\hline Estadual & & & 11 & 68,8 \\
\hline Particular & & & 1 & 6,3 \\
\hline
\end{tabular}

\begin{tabular}{|c|c|c|}
\hline \multicolumn{3}{|c|}{ Categoria Profissional } \\
\hline Enfermagem & 13 & 81,3 \\
\hline Medicina & 3 & 18,8 \\
\hline
\end{tabular}

\section{Categoria/Nível da Bolsa}

\begin{tabular}{l|l|l|l|l}
\hline $1 \mathrm{~B}$ & & & 2 & 12,5 \\
\hline $1 \mathrm{C}$ & & & 4 & 25,0 \\
\hline $1 \mathrm{D}$ & & & 2 & 12,5 \\
\hline 2 & & & 8 & 50,0 \\
\hline i Média ii Desvio Padrão
\end{tabular}

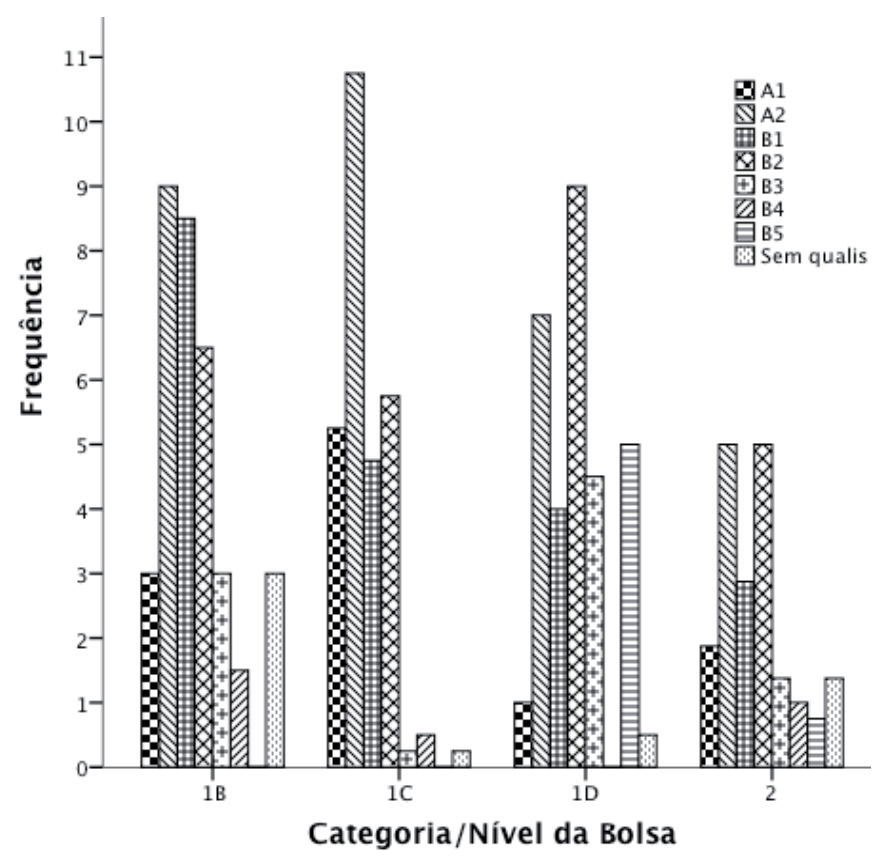

Figura 1: Qualis dos periódicos nos níveis/ categorias de bolsa dos bolsistas produtividade em pesquisa da área de Estomaterapia do CNPq no triênio 2010-2012. Teresina, PI, Brasil, 2014.

\section{DISCUSSÃO}

Este estudo apresenta resultados importantes sobre o perfil e a produção científica dos bolsistas produtividade em pesquisa do CNPq na área da Estomaterapia, especialidade jovem, mas em ascensão no Brasil ${ }^{10}$.

Para se tornar bolsista produtividade em pesquisa do $\mathrm{CNPq}$, alguns critérios devem ser atendidos como: possuir título de doutor ou perfil científico equivalente; ser brasileiro, ou estrangeiro com situação regular no País; dedicar-se às atividades de seu pedido de bolsa e poderá ser aposentado, desde que possua atividades acadêmicocientíficas oficialmente vinculadas às instituições de ensino e pesquisa ${ }^{11}$.

Em relação aos bolsistas deste estudo, a maioria, 12 (75\%), era do sexo feminino. Essa constatação está de acordo com a crescente presença feminina, verificada no período de 1990 a 2010 pelo CNPq, em pesquisas científicas. Isso pode ter ocorrido devido ao aumento de pessoas do sexo feminino em escolas e faculdades, se comparado ao sexo masculino ${ }^{12}$.

Houve maior concentração de bolsistas na região sudeste, 9 (56,3\%). Esta região concentra maior número de programas e cursos de Pós-graduação, com incentivo à produção científica. Resultados similares foram encontrados em estudos envolvendo diversas categorias profissionais da área da saúde ${ }^{13-15}$.

Dentre a categoria profissional, destacou-se a Enfermagem, 13 (81,3\%). Essa profissão se expande e se fortalece como ciência, tecnologia e inovação, que vem sendo consolidada pela ascensão e qualidade dos Programas de Pós-graduação stricto senso, aumento de publicações qualificadas e qualidade das revistas desta área. A expansão de cursos stricto senso representa o quantitativo de $1,7 \%$ do total de cursos de pós-graduação brasileiros, e 9,7\% desses são da área de Ciências da Saúde ${ }^{16}$.

A comunicação com a equipe médica tem papel importante no trabalho do estomaterapeuta. 0 planejamento pré operatório e a demarcação do local do estoma, por exemplo, é imprescindível para uma boa localização, o que permite a aderência do dispositivo e fácil visualização pelo paciente. A atuação multiprofissional, incluindo enfermeiro estomaterapeuta, assistente social, psicólogo, nutricionista, cirurgião, médico assistente, dentre outros visa uma boa atuação e comunicação com o paciente e a família, ao prestar assistência de qualidade e orientações que estimulem o autocuidado e a prevenção de complicações ${ }^{17}$.

Quanto à categoria de bolsa, predominou o nível PQ-2, 8 (50\%). Resultado semelhante foi encontrado em diferentes estudos do perfil de bolsistas produtividade em pesquisa do $\operatorname{CNPq}(55,7 \%)$ na área médica e $(48,3 \%)$ na saúde coletiva ${ }^{14,18}$. Vale ressaltar que as bolsas são oferecidas conforme atendimento aos critérios pré-estabelecidos pelo $\mathrm{CNPq}$ e a classificação, enquadramento e progressão 
do bolsista produtividade em pesquisa por categoria e nível é feita por Comitês de Assessoramento (CA). Os critérios de qualificação da bolsa são: a produção científica do candidato, formação de recursos humanos em nível de Pós-Graduação, contribuição científica e tecnológica para inovação, coordenação ou participação principal em projetos de pesquisa e participação em atividades editoriais e de gestão científica e administração de instituições e núcleos de excelência científica e tecnológica ${ }^{11}$.

O tempo médio de formação e de doutoramento foi respectivamente 33,3 e 16,4 anos. Existem requisitos mínimos para o enquadramento dos pesquisadores nos níveis/categorias das bolsas; primeiramente, pesquisadores da categoria 1 necessitam ter no mínimo oito anos de doutorado por ocasião da implementação da bolsa, já para o pesquisador 2, esse período se reduz a três anos, observando-se que a média de tempo de doutoramento dos pesquisadores se enquadrou ao preconizado pelo CNPq.

Em média, os bolsistas produtividade em pesquisa publicaram 24,7 (DP=14,9) artigos sobre temáticas gerais e 2,5 (DP= 5,3) na área de Estomaterapia. 0 aumento da produção científica se deve a expansão dos cursos de Pós-graduação, a ampliação da indexação de revistas em bases nacionais e internacionais e ao aumento dos índices censiométricos. Em 2005, a Enfermagem passou de 0,23\% da produção científica brasileira para 1,87\%, em 2010, com crescimento relativo de $713 \%$ àquele ocorrido nas Ciências Sociais e Medicina ${ }^{18}$.

A qualidade da produção intelectual dos pesquisadores é avaliada, segundo a Coordenação de Aperfeiçoamento de Pessoal de Nível Superior (CAPES), também conforme o Qualis do periódico publicado, que é dividido em oito estratos em ordem decrescente de valor: A1, A2, B1, B2, B3, B4, B5 e $\mathrm{C}^{19}$.

A comunidade científica busca continuamente publicação em periódicos indexados, sobretudo aqueles com Qualis A e B, nacionais e internacionais ${ }^{5}$. Os resultados deste estudo ratificaram essa afirmação, pois em média, os bolsistas produtividade em pesquisa publicaram 24,7 $(\mathrm{DP}=14,9)$ artigos sobre temáticas gerais e 2,5 $(\mathrm{DP}=5,3)$ na área de Estomaterapia em periódicos Qualis, sendo A2, $7,2(\mathrm{DP}=5,6) ; \mathrm{B} 2,5,8(\mathrm{DP}=4,9)$ e $\mathrm{B} 1,4,2$ (DP=4,9). Portanto, o produto das pesquisas dos bolsistas produtividade em pesquisa dessa área, na maioria das vezes, é publicado em periódicos científicos de boa qualidade. 0 aumento da publicação em periódicos científicos Qualis A1, A2 e B1 se deve às exigências atuais dos cursos de Pós-graduação stricto sensu ao reconhecimento da qualificação das revistas da Área de Enfermagem, bem como ao processo de internacionalização da ciência brasileira.

Entre os bolsistas produtividade em pesquisa da área de Estomaterapia das categorias de bolsa $1 \mathrm{C}$ e $1 \mathrm{~B}$ prevaleceu o Qualis A1 e A2. Estudos que utilizaram a mesma metodologia na área da Enfermagem e de medicina mostraram, para a mesma classe de pesquisadores, a predominância de artigos em periódicos Qualis B ${ }^{20,21}$. Vale lembrar que é importante relacionar os vieses da revista causados pelo produtivismo aos critérios não padronizados de avaliação editorial e, por isso, adotar mais critérios de avaliação na produção de cada pesquisador como, por exemplo, o índice $\mathrm{h}$, que é o impacto acadêmico pelo número de citações das publicações ${ }^{22}$.

O nível A corresponde a candidatos com excelência continuada na produção científica e na formação de recursos humanos, que liderem grupos de pesquisa consolidados. Os níveis B e C além de contribuírem para a produção de ciência, tecnologia e recursos humanos, contribuem nas atividades de pesquisa das suas instituições e/ou agências de fomento à pesquisa. Os pesquisadores com bolsa nível 2 são avaliados com ênfase nos trabalhos publicados e orientações, ambos referentes aos últimos cinco anos ${ }^{10}$.

\section{CONCLUSÃO}

O presente estudo descreveu o perfil e a produção científica dos bolsistas de Produtividade em Pesquisa do CNPq na área de estomaterapia no triênio 2010-2012. Identificou-se predomínio de bolsistas do sexo feminino, da área de Enfermagem, e bolsas PQ-2, concentrados na região sudeste. Em média, os bolsistas publicaram 24,7 $(\mathrm{DP}=14,9)$ artigos sobre temáticas gerais e 2,5 $(\mathrm{DP}=5,3)$ na área de Estomaterapia.

Tem-se como limitação do estudo a única fonte de coleta dos dados, que foi o Currículo Lattes. Não se verificou a veracidade de informações dos autores, pela limitação de sua natureza exploratória e restrições quanto às atualizações das informações feitas por eles. Vale lembrar, que o Currículo Lattes é considerado confiável para a caracterização dos pesquisadores uma vez que serve como critério nas avaliações de bolsas e na arrecadação de financiamento em editais de pesquisa.

Estudos dessa natureza são necessários para verificar o que tem sido produzido, além de permitir análise e reflexão sobre a qualidade das publicações. No entanto, ainda são escassos esses estudos no âmbito da Enfermagem, logo, é importante estimular pesquisas que tracem o perfil dos pesquisadores desta categoria profissional, não somente em Estomaterapia, mas nas mais diversas áreas temáticas.

\section{REFERÊNCIAS}

1. SCImago.SJR - SCImago Journal \& Country Rank. Retrieved on September 30, 2010. Available from: http://www.scimagojr. com.

2. Santos NCF, Candido LFO, Kuppens CL. Produtividade em pesquisa do CNPq: análise do perfil dos pesquisadores da Química. Quím. Nova (São Paulo). 2010;33(2):489-495.

3. Silva, LL. Estudo do perfil científico dos pesquisadores com bolsa de produtividade em pesquisa do CNPq que atuam no ensino de ciências e matemática. RBPEC. 2011; 11(3): 75-99. 
4. Oliveira EA; Pécoits-filho R, Quirino IG, Oliveira MC, Martelli DR, Lima LS, et al. Perfil e produção científica dos pesquisadores do CNPq nas áreas de Nefrologia e Urologia. J. Bras. Nefrol. 2011; 33(1): 31-37.

5. Oliveira MCL, Daniella RBM, Pinheiro SV, Miranda DM, Quirino IG, Leite BGL, et al. Perfil e produção cientifica dos pesquisadores do Conselho Nacional de Desenvolvimento Cientifico e Tecnologico na area de Pediatria. Rev. paul. pediatr. 2013;31(3):278-84.

6. Cavalcanti AL; Pereira DSA. Perfil do bolsista de produtividade em pesquisa do Conselho Nacional de Desenvolvimento Científico e Tecnológico (CNPq) na área de Odontologia. RBPG. 2008;5(9):67-88.

7. Souto RQ, Lacerda GS, Costa GMC, Cavalcanti AL, França ISX, Sousa FS. Caracterização dos pesquisadores bolsistas de produtividade do cnpq da área de enfermagem: estudo transversal. OBJN. 2012;11(2):261-73.

8. Associação Brasileira de Estomaterapia - SOBEST. Histórico. Acesso em: 17 jun 2014. Disponível em: http://www.sobest.org.br/index.php?option=com content \&view=article \&id=24\& Itemid $=47$

9. Oliveira Filho RS, Hochman B, Nahas FX, Ferreira LM. Fomento à publicação científica e proteção do conhecimento científico. Acta Cir. Bras. 2005;20(2):35-39.

10. Miranda LSG. A Importância da Consulta de Enfermagem de Estomaterapia na Qualidade de Vida da Pessoa Ostomizada na Comunidade [dissertação]. Vila Real: Universidade de Trásos-Montes e Alto Douro; 2013.

11. Conselho Nacional de Desenvolvimento Científico e Tecnológico (CNPq). Citado em: jun 2014. Disponível em:<http://www.cnpq.br/documents/10157/5f43cefd-7a9a4030-945e-4a0fa10a169a>.

12. Instituto Brasileiro de Geografia e Estatística - IBGE, 2010. Disponível em: <http://www.ibge.gov.br/>

13. Bezerra MLS, Neves EB. Perfil da Produção Científica em Saúde do Trabalhador. Saúde Soc. 2001;19(2):384-394.

14. Martelll-Junior H. Pesquisadores do Cnpq na área de Medicina: Comparação das áreas de atuação. Rev Assoc Med Bras. 2010;56(1):478-83.

15. Leite BD. Perfil dos Pesquisadores com Bolsa de Produtividade no Conselho Nacional de Desenvolvimento Científico e Tecnológico (CNPq) da Área da Educação Física. Motricidade. 2012; 8(3):90-98.

16. Coordenação de Aperfeiçoamento de Pessoal de Nível Superior-CAPES.Avaliação. Programas da Área da Enfermagem. [citado jun 2014]. Disponível em: <http://www.capes.gov.br/>.

17. Menezes LCG, Guedes MVC, Oliveira RM, Oliveira SKP, Meneses LST, Castro ME. Prática de autocuidado com estomizados: contribuições da teoria de Orem. Rev. Rene. 2013;14(2):301-10.

18. Santos SMC, Lima LS, Martelli DRB, Martelli-Junior R. Perfil dos pesquisadores da Saúde Coletiva no Conselho Nacional de Desenvolvimento Científico e Tecnológico. Physis. 2009; 19(3):761-775.

19. Coordenação de Aperfeiçoamento de Pessoal de Nível Superior - CAPES. Classificação da produção intelectual. Acesso em: 17 jun 2014. Disponível em: http://www.capes. gov.br/avaliacao/instrumentos-de-apoio/classificacao-daproducao-intelectual.
20. Mendes PHC; Martelli DRB, Souza WP, Filho SQ, Júnior HM. Perfil dos Pesquisadores Bolsistas de Produtividade Científica em Medicina no CNPq, Brasil. Rev. Bras. Educ. Med. 2010;34(4):535-541.

21. Santos WM, Padoin SMM, Lacerda MR, Gueterres EC. Perfil dos pesquisadores bolsistas de produtividade em pesquisa na área da enfermagem. Rev enferm UFPE on line. 2015;9(2):84450.

22. Weber JLA, Ramos CC, Mester A, Lindern D, Hörle KR, Souza CS, et al. Perfil dos pesquisadores bolsistas de produtividade científica em Psicologia do Conselho Nacional de Desenvolvimento Científico e Tecnológico. Estud. psicol. (Campinas). 2015:32(1):1-11. 